\title{
OPTIMISATION OF PRE-OPERATIVE ANAEMIA IN LOWER LIMB JOINT REPLACEMENT SURGERY: ASSESSING THE RATES OF ALLOGENIC BLOOD TRANSFUSION AND DURATION OF HOSPITAL STAY IN A DISTRICT GENERAL HOSPITAL
}

\author{
Sara Maki ${ }^{1}$, Neil Verma ${ }^{1}$, Winifred Morris ${ }^{1}$, and Jay Smith ${ }^{1}$ \\ ${ }^{1}$ Hillingdon Hospitals NHS Foundation Trust
}

September 11, 2020

\begin{abstract}
AIM: Orthopaedic procedures such as total hip and total knee replacements carry a significant risk of post-operative anaemia, necessitating allogenic blood transfusions (ABT), and an increased hospital length of stay. Our aim was to investigate whether the implementation of a local protocol designed to detect and treat pre-operative anaemia resulted in reduced ABT rates and a shorter duration of length of hospital stay (LOS). METHOD: We retrospectively audited 683 patients undergoing primary hip and knee replacements. We collated data for all patients about hospital length of stay and blood transfusions received. Both descriptive statistics and univariate analysis were performed. RESULTS: $21.6 \%$ of the cohort within the study who were anaemic at pre-operative clinic had a significantly increased median LOS of 2 days $(\mathrm{P}<0.001)$, and an increased packed red cell transfusion rate compared to non-anaemic patients $(26.1 \%$ vs $2.21 \%, \mathrm{P}<0.001)$. However, treatment of pre-operative anaemia did not show any significant difference in transfusion rates compared to patients who did not receive corrective treatment. The median LOS was higher by 1 day in the treated group compared to the non-treated cohort $(\mathrm{P}=0.005)$. CONCLUSION: There is significant evidence to suggest that pre-operative anaemia can increase LOS and increase the risk of requiring post-operative blood transfusions. However, anaemia should be regarded as a characteristic that can add to the outcome in a cumulative manner, as opposed to an isolated factor. Further research is needed on how to better manage pre-operative anaemia in order to improve patients' outcomes.
\end{abstract}

\section{What's already known about this topic?}

- Pre-operative anaemia and allogenic blood transfusion lead to poor post operative outcomes

- Pre-operative anaemia contributes to longer duration of hospital stay

\section{What does this article add?}

- This study has shown that there is no statistical difference between transfusion rates of anaemic patients optimised pre-operatively and anaemic patients who were inadvertently not.

- Hospital length of stay is in fact longer in anaemic patients who received corrective treatment compared to the anaemic cohort group who did not.

Keywords: anaemia, preoperative, arthroplasty, blood transfusion, outcome

\section{Background}


The World Health Organisation (WHO) originally defined anaemia based on haemoglobin $(\mathrm{Hb})$ levels as $<$ $130 \mathrm{~g} \mathrm{~L}-1$ for men and $<120 \mathrm{~g} \mathrm{~L}-1$ for non-pregnant women[6]. Based on this definition being widely used within protocols across the National Health Service (NHS), there have been large variations of the reported prevalence of anaemia within communities. Unfortunately, this definition does not account for other factors that may have important contributions to the effects of anaemia such as age, gender, ethnicity, symptoms of fatigue and the presence of cardiovascular disease, to name a few.

Two particular studies quote the prevalence of anaemia to be $20-25 \%$ of the population cohort presenting for major lower limb surgeries[7,8]. The relationship and impact of anaemia on surgical outcomes has been widely investigated to understand if improvements in the peri-operative period can lead to better results for patients. Historically, the initial studies began within cardiac surgery. From there, further studies were published looking into other specialities. Specifically delving into major lower limb orthopaedic surgery, a couple of studies suggested that allogenic blood transfusions in this cohort accounted for 8-10\% of the total donated blood transfusions within the UK[9, 10]. In addition to this, Kotze et al demonstrated that a patient's preoperative $\mathrm{Hb}$ concentration predicted the likelihood of the need to receive allogenic blood transfusions, as well as the hospital length of stay[7]. There is evidence to suggest that both anaemia and allogenic blood transfusions contribute in an accumulative method to risk factors for poor post-operative outcomes[11]. A retrospective study looking at 300,000 patients undergoing non-cardiac surgery identified that a preoperative haematocrit of less than $39 \%$ had a statistically significant increase in mortality at 30 days[12].

With an understanding of the impact of pre-operative anaemia the Network for Advancement of Transfusion Alternatives (NATA), constituted of a multidisciplinary panel of physicians, developed guidelines for identifying, evaluating and managing pre-operative anaemia in patients undergoing elective orthopaedic surgery[13]. These guidelines have been widely adapted across the UK as well as the implementation of enhanced recovery programmes to assist patients in achieving a satisfactory and better surgical outcome.

However, to best understand the management options, we must firstly understand the cause of the anaemia. A publication released through the AAGBI in 2015 suggested that the cause of anaemia could be categorised into 3 broad groups: nutrient deficiency anaemia, anaemia of chronic inflammation, and unexplained anaemia[14]. Nutrient deficiency anaemia includes deficiencies in iron (accounting for the largest cause of all anaemias at $16.6 \%[14])$, B12 and folate.

Anaemia of chronic inflammation (or chronic disease) incorporates conditions such as chronic kidney disease, autoimmune disorders, malignancy and inflammatory conditions. These are typically seen as normocytic and normochromic anaemia. There is often a crossover between these 2 groups of anaemias, typically identified by low iron stores due to the pathophysiology within the chronic inflammatory conditions. Hepcidin, a protein secreted by the liver, is key to regulating iron levels within the plasma. It inhibits iron absorption from the gut, as well as causing iron trapping within macrophages and liver cells. In chronic infection and inflammatory conditions, Hepcidin production is significantly increased which can lead to a 'functional' iron deficient anaemia[15]. The second mechanism by which anaemia of chronic inflammation occurs is by the reduction of erythropoiesis mediated by various cytokine pathways, including interleukin (IL)-6, as well as $\mathrm{TNF} \alpha$, IL- $1 \alpha$ and IL-1 $\beta$. There is also a third and final pathway to consider, in which cytokines cause a decrease in red cell survival and cell death. These mechanisms are also important to understand in the post-operative period because surgery will often blunt the body's response to anaemia through release of these inflammatory cytokines[16], and therefore, it could be expected that blood loss and intra-operative haemodilution could prolong the extent of post-operative anaemia.

The third category of unexplained anaemia includes those conditions that did not meet the criteria for the previous 2 groups based on detection and tests, and those who's conditions may not have been identified without further specialist tests (such as myeloma). Nevertheless, these patients can still be treated in spite of an exact cause not known.

Having understood the pathophysiology of anaemia, the NATA guidelines for the management of pre- 
operative anaemia (following detection and evaluation) concentrate on the correction of nutrient deficiency anaemia and anaemia of chronic inflammation. In the first instance, NATA recommends that nutritional deficiencies be treated, in particular, attempting to correct iron deficiency as it is the most common cause of anaemia. Traditionally, this has been through oral iron supplements. However, we know that these are not always well tolerated by patients as they can lead to gastrointestinal disturbances[17]. As an alternative, intravenous (IV) iron therapy has been recommended within the guidelines depending on the time scale as well as the intolerance of oral iron supplements. There have been studies in which IV iron supplementation reduced the requirements for allogenic blood transfusions in gynaecological cancer patients[18, 19].

The alternative stage recommended by NATA is the suggestion to stimulate erythropoiesis by erythropoiesisstimulating agents (ESA). The guidelines published first performed a meta-analysis of 41 studies looking into the use of ESA before recommending their concomitant use with iron therapy[13], which correlates in theory with the crossover in mechanism for the cause of anaemia in this group of patients.

\section{Method}

The Hillingdon Hospital NHS Foundation Trust perform on average over 600 elective primary lower limb joint replacement surgeries each year across 2 main sites (Hillingdon Hospital and Mount Vernon Hospital). This project originated as an audit to look into the compliance of a locally implemented protocol (Figure 1) in the pre-assessment clinic to identify, evaluate, and effectively manage anaemic patients booked for elective primary lower limb surgery.

Figure 1: 


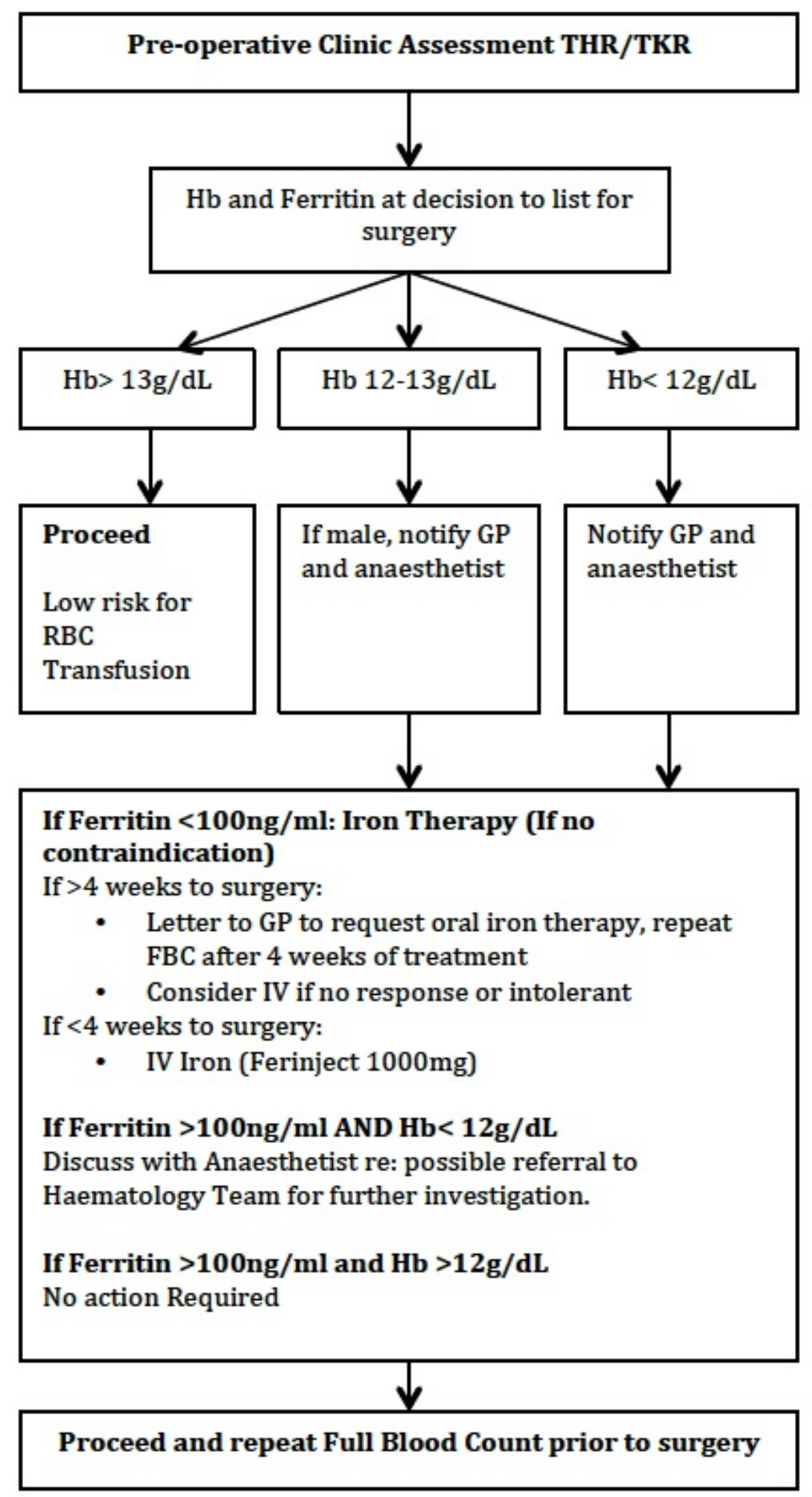

Figure 1 - The Hillingdon Hospitals NHS Foundation Trust Pre-operative anaemia optimisation protocol 
for elective total hip and total knee arthroplasties. THR: Total Hip Replacement; TKR: Total Knee Replacement; Hb: Haemoglobin; RBC: Red Blood Cells; GP: General Practitioner; FBC: Full Blood Count; IV: Intravenous.

Upon the decision to proceed for surgery, patients are referred to the pre-operative assessment clinic, where they undergo routine investigations (full blood count, urea and electrolytes, group and save, ECG) and an assessment of their medical co-morbidities. A protocol, based on the NATA guidelines previously discussed, was created specifically for patients undergoing elective primary hip or knee replacement surgery. This would be initiated at the pre-operative assessment clinic.

The haemoglobin and ferritin levels were evaluated, and patients who were identified as anaemic (as defined by the $\mathrm{WHO}$ criteria of $\mathrm{Hb}<12 \mathrm{~g} / \mathrm{dL}$ in non-pregnant women and $\mathrm{Hb}<13 \mathrm{~g} / \mathrm{dL}$ in men) and have a serum ferritin level of $<100 \mathrm{ng} / \mathrm{ml}$ receive oral iron therapy for four weeks through their GP. Where there is less than four weeks before the date of surgery, patients received intravenous iron, ferric carboxymaltose, instead.

These patients are then re-assessed to see if their anaemia has been optimised. If there has been no response to oral therapy, patients are then given intravenous iron therapy, provided that there are no contra-indications. In patients with a serum ferritin level of more than $100 \mathrm{ng} / \mathrm{mL}$ and a Haemoglobin of less than $12 \mathrm{~g} / \mathrm{dL}$, discussion with the haematology team is recommended to further investigate and address the cause of anaemia.

We conducted a retrospective audit of patients who had undergone elective primary total hip or total knee arthroplasty in 2016. A patient database was obtained from the clinical coding department. We initially analysed all patients' blood results using the Trust's electronic blood test reporting system. Once the anaemic patients were identified from their initial pre-operative assessment bloods, we used their inpatient clinical notes, electronic discharge summaries and electronic GP records to determine which patients received pre-operative anaemia correction treatment.

683 patients were included in the audit from the database that had undergone surgery between January 1st 2016 and December 31st 2016. From the same database, 21 patients were excluded for a variety of reasons - 18 patients had revision arthroplasty surgeries, 1 patient had bilateral joint replacement surgery, 1 patient was receiving blood transfusions on a regular basis prior to surgery for a complex haematological condition, and 1 patient had no discharge summary. From this, we established that 138 patients were identified as being anaemic at pre-operative assessment clinic (20.2\% of the cohort population).

Only 25 of the 138 anaemic patients were correctly identified and managed with regards to their anaemia and the pre-operative assessment protocol (18.1\%).

Following on from the results of the audit, we decided to examine what impact anaemia and the subsequent management protocol had on hospital length of stay and blood transfusion requirement. This was done by 2 methods: For hospital length of stay we obtained the information from patients' electronic discharge summaries. For blood transfusions, we used the Trust's electronic blood test and investigation reporting system to determine how many units of blood were issued for each patient (if any) and cross referenced with their electronic discharge summaries. The length of stay and blood transfusion rates were calculated for both anaemic and non-anaemic patients.

\section{Statistical analysis}

Statistical analysis was performed using $\mathrm{R}$ v 3.5.2. Descriptive statistics were performed using percentage (\%) for categorical variables and using median and interquartile range [IQR] for continuous variables since the LOS was right-skewed (non-normal). Consequently, univariate analysis was performed using Mann-Whitney and Kruskal-Wallis test for non-normal dependent variables (e.g. LOS) when the independent variable had two and three levels, respectively. Chi-square test of independence was used to assess the association between anaemia and the need for transfusion.

Negative binomial regression (NBR) was used to assess the association between anaemia and LOS. Age, 
gender, and site of surgery (knee vs. hip) were included as covariates to the model as a separate interest.

\section{Results}

A total of 683 patients underwent either a primary total knee replacement or a primary total hip replacement. From the descriptive statistics in the table below (Table 1), the percentage of females $(\mathrm{n}=419,61.3 \%)$ was higher compared to males $(\mathrm{n}=264,38.7 \%)$ in the study sample; knee procedures represented $63.7 \%(\mathrm{n}=$ $435)$ of the total procedures performed. Anaemia was present in 138 patients $(20.2 \%)$ and transfusion was performed in 48 patients $(7.05 \%)$ of the total study sample. The median length of stay was 4 [IQR 3 - 7] days.

Table 1. Descriptive statistics for the study sample

Summary descriptives table

[ALL] $\mathrm{N}=683$

Gender:

Female

$419(61.3 \%)$

Male

$264(38.7 \%)$

Age group:

$<65$

$217(31.8 \%)$

65-75

$259(37.9 \%)$

$>75$

$207(30.3 \%)$

Site:

Hip

$248(36.3 \%)$

Knee

$435(63.7 \%)$

Anaemic:

No

$545(79.8 \%)$

Yes

$138(20.2 \%)$

Transfusions performed:

No

$633(93.0 \%)$ 
Yes

$48(7.05 \%)$

Length of stay

4.00 [3.00;7.00]

Statistical analysis results in Table 2 showed that the median length of stay was significantly higher in anaemic patients compared to non-anaemic patients $(6$ days vs. 4 days, $\mathrm{P}<0.001)$. As expected, allogenic blood transfusions were given to a significantly higher number of anaemic patients compared to non-anaemic patients $(26.1 \%$ vs. $2.21 \%, \mathrm{P}<0.001)$.

Table 2. Univariate analysis by anaemia

Summary descriptives table by groups of 'anaemic'

Non-anaemics $\mathrm{N}=545$

Anaemics $\mathrm{N}=138$

$\mathrm{P}$

Gender:

0.253

Female

$328(60.2 \%)$

$91(65.9 \%)$

Male

$217(39.8 \%)$

$47(34.1 \%)$

Age group:

$<0.001$

$<65$

$193(35.4 \%)$

$24(17.4 \%)$

65-75

$206(37.8 \%)$

$53(38.4 \%)$

$>75$

$146(26.8 \%)$

$61(44.2 \%)$

Site:

0.007

Hip 
$212(38.9 \%)$

36 (26.1\%)

Knee

$333(61.1 \%)$

$102(73.9 \%)$

Transfusion:

$<0.001$

No

$531(97.8 \%)$

$102(73.9 \%)$

Yes

$12(2.21 \%)$

$36(26.1 \%)$

LOS

$4.00[3.00 ; 6.00]$

6.00 [4.00;8.00]

$<0.001$

Statistical analysis was performed using Mann-Whitney test for length of stay and Chi-square test for independence for all the remaining comparisons

Statistical analysis was performed using Mann-Whitney test for length of stay and Chi-square test for independence for all the remaining comparisons

Statistical analysis was performed using Mann-Whitney test for length of stay and Chi-square test for independence for all the remaining comparisons

Statistical analysis was performed using Mann-Whitney test for length of stay and Chi-square test for independence for all the remaining comparisons

The need for blood transfusion was significantly different between the three groups $(\mathrm{P}<0.001)$. Pairwise comparisons (Table 3) showed that the percentage of patients who required transfusion was lower in nonanaemic patients compared to correctly treated patients $(2.2 \%$ vs. $24 \%, \mathrm{P}<0.001)$ and incorrectly treated patients $(2.2$ vs. $26.5 \%, \mathrm{P}<0.001)$ but not between correctly and incorrectly managed anaemic patients $(\mathrm{P}$ $>0.05)$.

Table 3. Pairwise comparisons between anaemics, correctly and incorrectly treated groups

Summary descriptives table by groups of 'cat'

Group

Group

Group

P for pairwise comparisons\#

$\mathrm{P}$ for pairwise comparisons\# 
P for pairwise comparisons\#

Group 1 (G1) N=545 Non-anaemic

Group 2 (G2) N=25 Anaemic Correct protocol

Group 3 (G3) N=113 Anaemic Incorrect protocol

$\mathrm{P}^{*}$

G1 vs. G2

G1 vs. G3

G2 vs. G3

\section{Transfusion}

$<0.001$

$<0.001$

$<0.001$

0.991

No

$533(97.8 \%)$

$19(76.0 \%)$

$83(73.5 \%)$

Yes

$12(2.20 \%)$

$6(24.0 \%)$

$30(26.5 \%)$

\section{LOS}

$4.00[3.00 ; 6.00]$

$7.00[6.00 ; 10.0]$

$6.00[4.00 ; 8.00]$

$<0.001$

$<0.001$

$<0.001$

0.005

* Statistical significance was determined by using Kruskal-Wallis test for LOS and Chi-square test of independence for transfusion rates. \#Statistical analysis for pairwise comparisons was performed using MannWhitney test for LOS and Chi-square test of independence for transfusion rates.

* Statistical significance was determined by using Kruskal-Wallis test for LOS and Chi-square test of independence for transfusion rates. \#Statistical analysis for pairwise comparisons was performed using MannWhitney test for LOS and Chi-square test of independence for transfusion rates. 
* Statistical significance was determined by using Kruskal-Wallis test for LOS and Chi-square test of independence for transfusion rates. \#Statistical analysis for pairwise comparisons was performed using MannWhitney test for LOS and Chi-square test of independence for transfusion rates.

* Statistical significance was determined by using Kruskal-Wallis test for LOS and Chi-square test of independence for transfusion rates. \#Statistical analysis for pairwise comparisons was performed using MannWhitney test for LOS and Chi-square test of independence for transfusion rates.

* Statistical significance was determined by using Kruskal-Wallis test for LOS and Chi-square test of independence for transfusion rates. \#Statistical analysis for pairwise comparisons was performed using MannWhitney test for LOS and Chi-square test of independence for transfusion rates.

* Statistical significance was determined by using Kruskal-Wallis test for LOS and Chi-square test of independence for transfusion rates. \#Statistical analysis for pairwise comparisons was performed using MannWhitney test for LOS and Chi-square test of independence for transfusion rates.

* Statistical significance was determined by using Kruskal-Wallis test for LOS and Chi-square test of independence for transfusion rates. \#Statistical analysis for pairwise comparisons was performed using MannWhitney test for LOS and Chi-square test of independence for transfusion rates.

* Statistical significance was determined by using Kruskal-Wallis test for LOS and Chi-square test of independence for transfusion rates. \#Statistical analysis for pairwise comparisons was performed using MannWhitney test for LOS and Chi-square test of independence for transfusion rates.

Using negative binomial regression analysis as illustrated in table 4 below, we are able to look at independent variables to predict their impact on LOS.

Table 4. Negative binomial regression analysis for predictors of Length of Stay

\begin{tabular}{|c|c|c|c|c|}
\hline Predictors & $\begin{array}{l}\text { Incidence Rate } \\
\text { Ratios (IRR) }\end{array}$ & Lower $95 \%$ CI & Upper $95 \%$ CI & $p$ \\
\hline Non-anaemia & 0.97 & 0.88 & 1.08 & 0.59 \\
\hline $\begin{array}{l}\text { Anaemia } \\
\text { (correctly } \\
\text { treated) }\end{array}$ & 1.53 & 1.26 & 1.87 & $<0.001$ \\
\hline Site (Knee) & 0.9 & 0.79 & 1.01 & 0.08 \\
\hline Age $(65-75)$ & 1.13 & 1.04 & 1.23 & 0.004 \\
\hline Age $(>75)$ & 1.45 & 1.33 & 1.59 & $<0.001$ \\
\hline Gender (Male) & 0.91 & 0.85 & 0.98 & 0.011 \\
\hline Transfusion (Yes) & 1.49 & 1.28 & 1.73 & $<0.001$ \\
\hline *Incorrectly & *Incorrectly & *Incorrectly & *Incorrectly & *Incorrectly \\
\hline treated anaemic & treated anaemic & treated anaemic & treated anaemic & treated anaemic \\
\hline patients were & patients were & patients were & patients were & patients were \\
\hline used as the & used as the & used as the & used as the & used as the \\
\hline control group & control group & control group & control group & control group \\
\hline
\end{tabular}

The expected LOS does appear to be lower in non-anaemic patients. However, this was not proven to be statistically significantly when compared to patients in whom the anaemia was not corrected $(I R R=0.97, \mathrm{P}$ $>0.05)$. However, using the same comparison group the expected LOS is $53 \%$ higher in participants whose anaemia was corrected $(\mathrm{IRR}=1.53, \mathrm{P}<0.001)$.

Site of surgery showed a statistically significant association with the expected LOS $(\operatorname{IRR}=0.9, \mathrm{P}=0.05)$, which indicates that the expected LOS was $10 \%$ lower among participants who underwent knee surgery compared to participants who underwent hip surgery. Age was also a significant predictor of LOS. The 
expected LOS in patients aged $65-75$ and $>75$ was $13 \%(\mathrm{P}<0.05)$ and $45 \%(\mathrm{P}<0.001)$ higher compared to patients aged $<65$ years, respectively. Gender showed a statistically significant association with LOS $(\mathrm{IRR}=0.92, \mathrm{P}<0.05)$ where the expected LOS was $8 \%$ lower in men compared to women. Finally, blood transfusion significantly predicted a $49 \%$ increase in the expected LOS $(\operatorname{IRR}=1.49, \mathrm{P}<0.001)$.

\section{Discussion}

\section{The impact of hospital length of stay}

Understanding the relationship between anaemia and surgical outcomes is a complex model to comprehend with the multiple covariates that come associated with low haemoglobin levels. For example, Table 2 shows the increase incidence of anaemia with increasing age $(\mathrm{P}<0.001)$, but it is possible that increasing age alone could bring its own challenges when considering co-morbidities (such as coronary artery disease), frailty and social circumstances. Consequently, when interpreting the results, it is perhaps fair to suggest that the relationships be considered more as an additive contribution rather than a stand-alone single causative characteristic[8].

Considering the LOS in this study, the evidence concludes that non-anaemic patients had a statistically significant reduced hospital length of stay than the anaemic patients undergoing major lower limb surgery (primary hip and knee replacements). Studies have shown that an enhanced recovery programme (ERP) can reduce length of stay[20,21], and many hospitals within the UK now deploy an enhanced recovery programme which include a multidisciplinary approach to the patient's overall care. This includes pre-operative assessment to optimise patients medically, looking at anaesthetic techniques to reduce post-operative pain, early mobilisation with the aid of physiotherapists, and surgical techniques. This study focuses on the pre-optimisation of anaemia, just one significant aspect of the ERP given the population that present with anaemia in this cohort.

However, as stated previously, this particular characteristic cannot be taken in isolation, and must be considered as an additive variable. Studies have shown that anaesthetic technique for lower limb joint replacements can alter the patient experience and the surgical outcomes as well[21]. For example, the use of general anaesthesia has shown to increase the requirements of post-operative opiate use for analgesia[22], which can lead to an increase incidence of post-operative nausea and vomiting as well as a reluctance and difficulty in early mobilisation due to pain - both of which can increase hospital length of stay[23]. In this study, we did not examine the type of anaesthesia used in all patients, the type of opiate used intrathecally for regional techniques (short acting fentanyl vs longer acting diamorphine) and the dose it was used in, and nor did we evaluate the concomitant use of non-steroidal anti-inflammatory drugs (NSAIDs) which may often be omitted in patients whose conditions may contraindicate its use. Likewise, taking into consideration surgical techniques, we did not review the use of local anaesthetic infiltration into the surgical site as recommended by the Hillingdon and Mount Vernon rapid recovery programme anaesthesia and analgesia guideline published in 2014 (Figure 2).

Figure 2: 


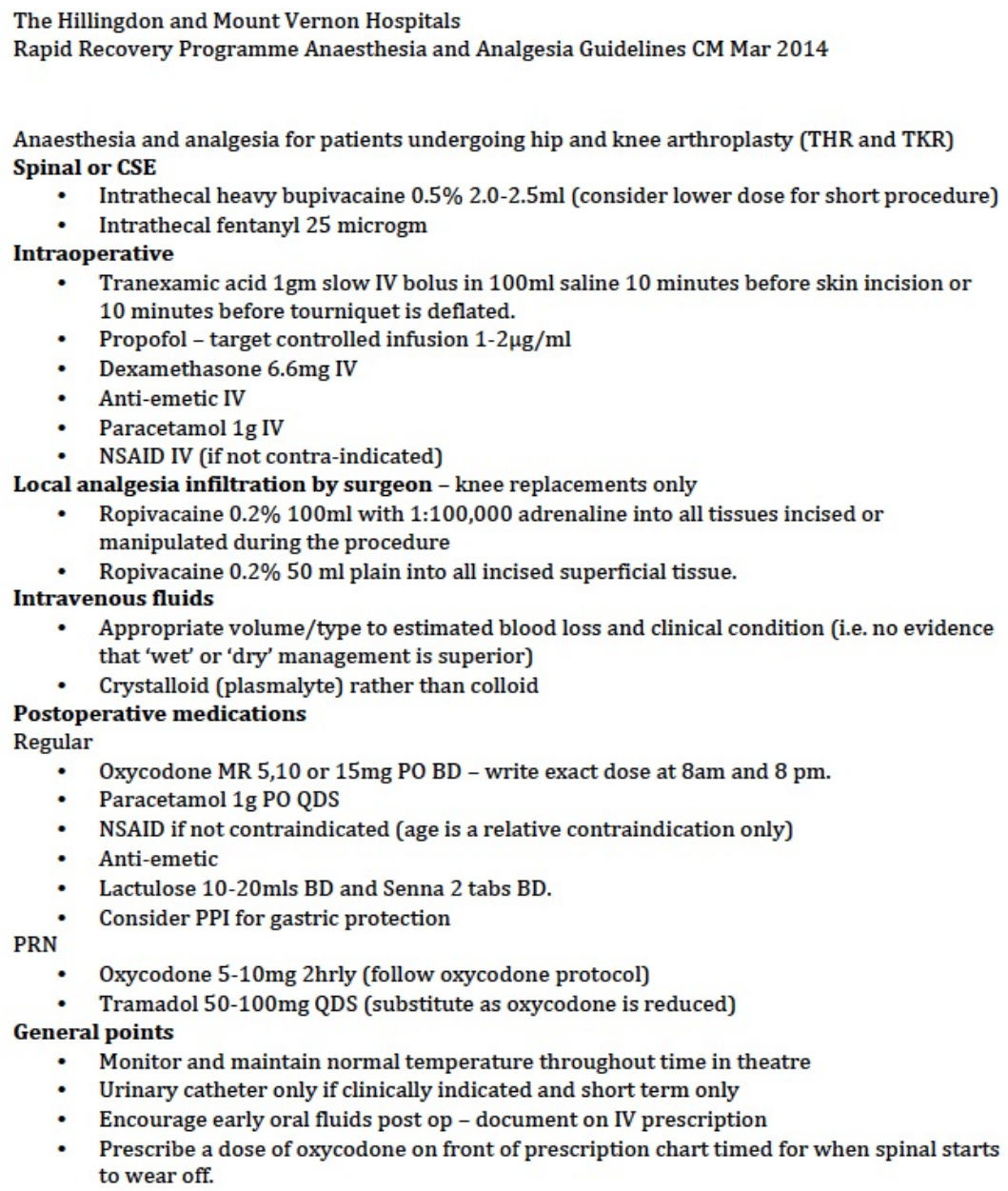

Figure 2 - The Hillingdon and Mount Vernon Hospitals Rapid Recovery Programme Anaesthesia and Analgesia Guidelines

Interestingly, the study does raise questions with regards to the management of anaemic patients. Anaemic patients who did not have their anaemia corrected had a statistically significant reduced LOS than anaemic patients who were managed according to the protocol (median LOS 6 days vs 7 days, $\mathrm{P}=0.005$ ). Negative binomial regression analysis also signifies that managing anaemia according to the protocol does have a predictably longer LOS as demonstrated in Table $4(\mathrm{P}<0.001)$, thus supporting the evidence seen. This would suggest that the current management protocol is not effective. NATA guidelines had suggested that as well as iron therapy, erythropoietin injections could also be considered as part of the management protocol[8, 13] to manage both iron deficiency anaemia and anaemia of chronic disease. It is also worth noting that oral iron therapy is not always well tolerated by patients (with the side effects of gastrointestinal disturbances) and hence, patient compliance may play a factor in the results seen. Nevertheless, with the small sample size of 25 anaemic patients receiving pre-operative treatment, and without examining the anaesthetic technique used (general vs regional anaesthesia) it is difficult to speculate on the cause for this difference seen. However, the statistics do show a significant difference and it is one that could benefit from further studies to understand this better. It is also worth taking into consideration complications unrelated to anaemia and the surgery which could have contributed to some patients requiring a longer length of stay, for example, post-operative hospital acquired pneumonia or myocardial infarction. These are not reflected separately in the results, and 
although they may be comparably low in numbers, they may skew the data in a small cohort.

\section{The impact on allogenic blood transfusion rates}

Correspondingly, allogenic blood transfusion rates also showed a predictable pattern with the incidence of transfusion being significantly less in non-anaemic patients than compared with anaemic patients $(2.2 \mathrm{vs}$ $26.1 \%, \mathrm{P}<0.001)$. However, there was no significance identified for transfusion rates between the 2 groups of anaemic patients (those treated pre-operatively vs those who did not receive pre-operative treatment). Similarly to the possible theory behind the LOS in both of these groups, questions may be asked of whether the use of erythropoietin injections to help stimulate growth of red blood cells as well as patient compliance to oral iron therapy contribute to these results. However, there are other significant factors that have not been taken into consideration when examining the transfusion rates. For instance, we did not account for the blood loss recorded for each patient. Primary knee replacement surgery commonly makes use of a tourniquet to reduce blood loss and assist in the visualisation of the surgical field - a surgical technique not possible with hip arthroplasties. It may be worth in the future considering the difference between the 2 sites of surgery and looking at each individual's outcomes. The guideline illustrated in figure 2 above, also advocates the use of tranexamic acid, another factor we have not included in our analysis.

It is worth considering in this section transfusion threshold. The decision to give allogenic blood transfusions often rests on checking Hb levels, and considering a patient's existing medical co-morbidities. When authorising blood transfusions, a risk versus benefit model must be considered as there are potential clinical incentives as well as cost benefits to be gained from avoiding it if it is not necessary[14], with each unit of packed red cell costing the Trust £133.22. There have been a variety of studies looking at strategies of liberal versus restrictive blood transfusions, and their outcome[24, 25]. The TRICC trial suggested that there was an equivalent benefit of using a restrictive strategy in ABTs within the elderly, but possibly a superior benefit in the under $55 \mathrm{~s}[26]$. Another study involving elderly patients with hip fractures suggested that liberal use of ABTs in patients with pre-existing risk factors for cardiac disease also showed no evidence of differences between the 2 groups[27].

Guidelines for anaesthetists within the UK were first published in 2008, followed by an update in 2016 which are more suggestive of using a restrictive ABT strategy to treat the anaemia in perspective to the surgery as opposed to their pre-operative haemoglobin level[28]. However, there is a loop hole to this guideline in which it suggests that transfusions maybe considered in patients who are symptomatic. This margin inevitably leads to a grey area in which patients and clinicians perceive the need for ABTs. This inconsistency in clinical management is one factor that may explain the unexpected results when analysing the 2 groups within the anaemia arm of the study. Another factor to also consider is that within the data reviewed, there was no specific mention of whether autologous blood transfusions were given via use of a cell salvage machine, thus reducing the possible need for allogenic blood transfusions.

\section{Conclusion}

With anaemia present in a significantly large proportion of the population presenting for lower limb joint replacement surgery, the results of this study signify a difference in length of hospital stay and transfusion rates between non-anaemic patients and anaemic patients. However, this study has shown that there is no statistical difference between transfusion rates of anaemic patients optimised pre-operatively and anaemic patients who were inadvertently not. Furthermore, the hospital length of stay is in fact longer in anaemic patients who received corrective treatment compared to the anaemic cohort group who did not.

It is important to consider the impact of anaemia as a contributing variable to the outcome as opposed to a stand-alone characteristic, particularly as there are limitations to this study. These include taking into consideration other relevant and important factors such as peri-operative blood loss, patients' co-morbidities, anaesthetic techniques and transfusion thresholds for individual patients.

Nevertheless, although patients should ultimately be managed on an individual basis in contrast to treating their numbers, more research would be beneficial in ascertaining on how to best manage anaemic patients 
to improve and match their outcome to their non-anaemic counterparts, as well as be able to bring the cost down for the NHS.

\section{Acknowledgments}

This project is the result of work supported with resources and the use of facilities at The Hillingdon Hospital NHS Foundation Trust.

\section{Author Disclosures}

The authors report no actual or potential conflicts of interest with regard to this article.

\section{References}

1. Bierbaum B, Callaghan J, et al., (1999) An Analysis of Blood Management in Patients Having a Total Hip or Knee Arthroplasty. The Journal of Bone \& Joint Surgery, 81(1): p 2-10.

2. Klein H., (1995) Allogeneic transfusion risks in the surgical patient. The American Journal of Surgery, 170(6): p. 21-26.

3. Marcucci C, Madjdpour C, et al., (2004) Allogenic blood transfusions: benefit, risks and clinical indications in countries with a low or high human development index. British Medical Bulletin, 70: p. $15-28$

4. Gombotz, H, Rehak P, et al., (2007) Blood use in elective surgery: the Austrian benchmark study. Transfusion, 47(8): p. 1468-1480.

5. Salido J, Marín L, et al., (2002) Preoperative Hemoglobin Levels and the Need for Transfusion After Prosthetic Hip and Knee Surgery. The Journal of Bone and Joint Surgery-American Volume, 84(2): p. 216-220.

6. Blanc B, Finch CA, et al., (1968) Nutritional anaemias. Report of a WHO Scientific Group. WHO Technical Report Series, 405: p. 1-40.

7. Kotzé A, Carter LA, et al., (2012) Effect of a patient blood management programme on preoperative anaemia, transfusion rate, and outcome after primary hip or knee arthroplasty: a quality improvement cycle. British Journal of Anaethesia, 108: p. 943-955.

8. Saleh E, McClelland DB, et al., (2007) Prevalence of anaemia before major joint arthroplasty and the potential impact of preoperative investigation and correction on perioperative blood transfusions. British Journal of Anaesthesia, 99: p. 801-808

9. Spahn DR., (2010) Anemia and patient blood management in hip and knee surgery. Anesthesiology, 113: p. 482-9

10. Husted H, Holm G, et al., (2008) Predictors of length of stay and patient satisfaction after hip and knee replacement surgery: fast-track experience in 712 patients. Acta Orthopaedica, 79: p. 168-73

11. Pederson AB, Mehnert F, et al., (2009) Allogenic blood transfusion and prognosis following total hip replacement: a population-based follow up story. BMC Musculoskeletal Disorders, 29: p. 16-26

12. Wu WC, Schifftner TL, et al., (2007) Preoperative hematocrit levels and postoperative outcomes in older patients undergoing non-cardiac surgery. JAMA, 297: p. 2481-8

13. Goodnough LT, Maniatis A, et al., (2011) Detection, evaluation, and management of preoperative anaemia in the elective orthopaedic surgical patient: NATA guidelines. British Journal of Anaesthesia, 106: p. 13-22.

14. Mannings A, Moppett I., (2015) Transfusion requirements and the older person. AAGBI Core Topics in Anaesthesia, 7: p. 80-90

15. Cullis JO., (2011) Diagnosis and management of anaemia of chronic disease: current status. British Journal of Haematology, 154: p. 289-300

16. Weiss G, Goodnough LT., (2005) Anaemia of chronic disease. The New England Journal of Medicine, 352: p. 1011-23

17. Tolkien, Z., Stecher, L., et al., (2015) Ferrous Sulfate Supplementation Causes Significant Gastrointestinal Side-Effects in Adults: A Systematic Review and Meta-Analysis. PLOS ONE, 10(2): p.e0117383.

18. Kim YT, Kim SW, et al., (2007) Effect of intravenously administered iron sucrose on the prevention 
of anaemia in the cervical cancer patients treated with concurrent chemoradiotherapy. Gynecologic Oncology, 105: p. 199-204

19. Dangsuwan P, Manchana T., (2010) Blood transfusion reduction with intravenous iron in gynecologic patients receiving chemotherapy. Gynecolgic Oncology, 116: p. 522-525

20. Tucker A, McCusker D, et al., (2016) Orthopaedic enhanced recovery programme for elective hip and knee arthroplasty - could a regional programme be beneficial? The Ulster Medical Journal, 85(2): p. 86-91

21. Hu ZC, He LJ, et al., (2019) An enhanced recovery after surgery program in orthopedic surgery: a systematic review and meta-analysis. Journal of Orthopaedic Surgery and Research, 14(1): p. 77

22. Gonano C, Leitgeb U, et al., (2006) Spinal Versus General Anesthesia for Orthopedic Surgery: Anesthesia Drug and Supply Costs. Anesthesia \& Analgesia, 102(2): p. 524-529

23. Johnson RL, Kopp SL, et al., (2016) Neuraxial vs general anaesthesia for total hip and total knee arthroplasty: a systematic review of comparative effectiveness research. British Journal of Anaesthesia, 116(2): p. 163-76

24. Al-Turki AA, Al-Araifi AK, et al., (2017) Predictors of blood transfusion following total knee replacement at a tertiary care center in Central Saudi Arabia. Saudi Medical Journal, 38(6): p. 598-603

25. Carson JL, Carless PA, Herbert PC., (2012) Transfusion thresholds and other strategies for guiding allogeneic red blood cell transfusion. Cochrane Database of Systematic Reviews, 4: Art no. CD002042.

26. Herbert P, Wells G, Blajchman M, et al., (1999) A multicenter, randomized, controlled clinical trial of transfusion requirements in critical care. The New England Journal of Medicine, 340: p. 409-417

27. Carson JL, Duff A, Berlin JA, et al., (2011) Liberal or restrictive transfusion in high-risk patients after hip surgery. The New England Journal of Medicine, 365: p. 2453-2462

28. Association of Anaesthetists of Great Britain and Ireland. (2016) AAGBI guidelines: the use of blood components and their alternatives. Anaesthesia, 71: p. 829-842

29. Dunne J, Malone D, et al., (2002) Perioperative Anemia: An Independent Risk Factor for Infection, Mortality, and Resource Utilization in Surgery. Journal of Surgical Research, 102(2): p. 237-244.

30. Rogers B, Cowie A, et al., (2008) Identification and treatment of anaemia in patients awaiting hip replacement. Annals of the Royal College of Surgeons of England, 90: p. 504-507 